\title{
MBIR 3D Reconstruction Method Effectively Minimizes Missing Wedge Artifacts and Restores Missing Information in Cryo-electron Tomography
}

\author{
Rui Yan ${ }^{1}$, Singanallur Venkatakrishnan ${ }^{2}$, Jun Liu ${ }^{3}$, Charles Bouman ${ }^{4}$ and Wen Jiang ${ }^{4}$
}

${ }^{1}$ Howard Hughes Medical Institute, Ashburn, Virginia, United States, ${ }^{2}$ Oak Ridge National Laboratory, Oak Ridge, Tennessee, United States, ${ }^{3}$ Yale University School of Medicine, West Haven, Connecticut, United States, ${ }^{4}$ Purdue University, West Lafayette, Indiana, United States

Cryo-electron tomography (cryo-ET) has emerged as an essential technique that allows us to reveal macromolecular complexes and cellular architecture in their near-native states (Lucić et al., 2005). In cryo-ET, the biological samples are imaged as they are tilted at different projection angles around a tilt axis, resulting in 2D tilt series that can be reconstructed to 3D tomograms (Herman, 2009). However, due to the radiation damage and the limited tilt range, cryo-ET suffers from low contrast and missing wedge artifacts, which deteriorates the resolution of 3D reconstruction and weakens the interpretability of the reconstructed tomogram (Herman, 2009; Lucić et al., 2005). To address these challenges, we introduced the Model-Based Iterative Reconstruction (MBIR) method (Venkatakrishnan et al., 2015; Yan et al., 2019) for tomographic reconstruction and demonstrated the advantages of MBIR in contrast improvement, missing wedge artifacts reduction, missing information restoration, and subtomogram averaging compared with other reconstruction approaches, including Weighted Back Projection (WBP) (Radermacher, 1992), Simultaneous Iterative Reconstruction Technique (SIRT) (Gilbert, 1972), and Iterative Compressed-sensing Optimized Non-uniform fast Fourier transform reconstruction (ICON) (Deng et al., 2016).

We first evaluated the reconstruction quality of MBIR on a cryo-ET dataset (EMPIAR-10045) by visually examining the missing wedge artifacts of gold markers. The missing wedge problem can be indicated by the elongation of gold markers, halos and streaking artifacts in the adjacent region in different slice views of the tomograms. As shown in Fig. 1A-D, it is clear that MBIR (Fig. 1D) can eliminate the halos and streaking artifacts, reduce the elongation of gold markers and enhance the contrast of the biological structures, compared with the performance of other reconstruction methods.

Next, we used the leave-one-out FRC method (Cardone et al., 2005; Yan et al., 2019) to assess the MBIR restoration of the missing information. In this method, the FRC is calculated between the raw tilt image and the corresponding reprojected image from a tomogram reconstructed without this tilt and used as a quantitative evaluation of the quality of the recovered information. In Fig. 1E, it is evident that the gold marker in MBIR reprojection of low and high tilt angles (the last column in Fig. 1E) is round, sharp-edged without discernible distortion or blurring, which is nearly identical to the original tilted image (the first column in Fig. 1E). Furthermore, the FRC curves of MBIR in Fig. 1F-G exhibit a significantly higher correlation between the reprojection and the original tilt, validating MBIR's capability to restore the missing information.

We further studied if MBIR is able to improve the map quality of subtomogram averages. Fig. 2A, B and E show the averaged subtomograms of WBP, MBIR maps and their "gold standard" FSC curves, respectively. However, it can be seen that the FSCs do not drop to zero, implying the "gold standard" FSC curves from the half maps may not be a reliable measurement of resolution for this dataset. Moreover, we calculated the FSCs between the averaged subtomograms and additional reference maps at higher reported resolutions, including EMD-3228 at $13 \AA$ resolution (Fig. 2C) (Bharat et al., 2015) and EMD-8799 at 
7.8 Å resolution (Fig. 2D) (Himes and Zhang, 2018). The MBIR's FSCs (green line in Fig. 2F-G) show significant overall improvement compared with WBP's FSCs (blue line in Fig. 2F-G), confirming the better quality of the subtomogram average from MBIR than WBP.

Our results have effectively demonstrated the excellent performance of MBIR in contrast improvement, missing wedge artifacts reduction, missing information restoration, and subtomogram averaging. The clear benefits of MBIR should help achieve better quality reconstruction, facilitate further visualization, such as biological feature interpretation, structure segmentation, and ultimately push cryo-ET to higher resolution (Yan et al., 2019).

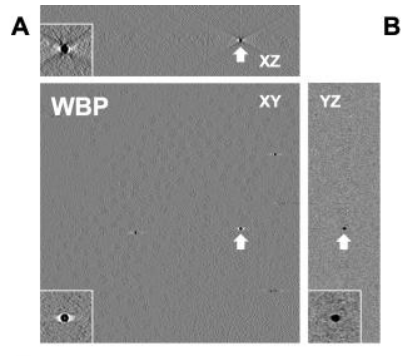

E
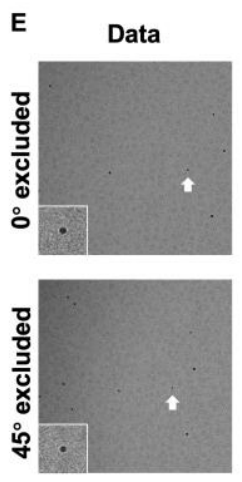

WBP
B
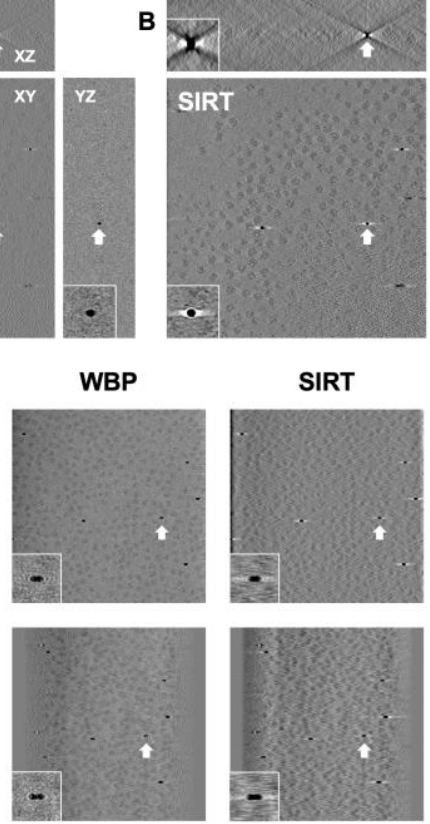

SIRT
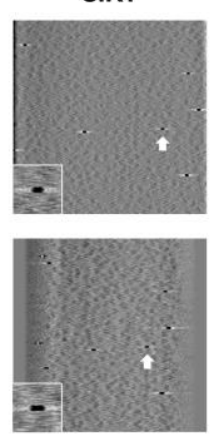

c
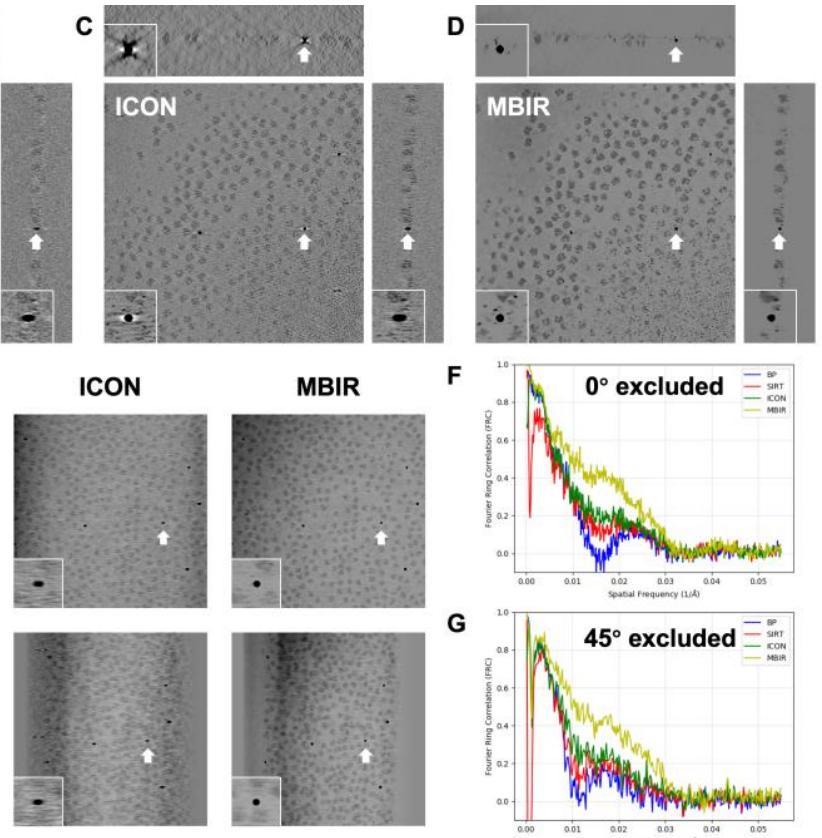

Figure 1. Comparison of tomograms and missing information restoration from an experimental cryo-ET dataset (EMPIAR-10045). (A-D) Tomograms reconstructed by WBP (A), SIRT (B), ICON (C) and MBIR (D) methods. The three planes for each method represent the XY-slice (middle plane), XZ-slice (top plane) and YZ-slice (right plane) of the tomogram intersecting at the same gold marker. (E) Comparison of reprojections at two tilt angles $\left(0^{\circ}\right.$ in the first row and $45^{\circ}$ in the second row) using the tomograms generated by different reconstruction techniques without the corresponding tilt. The images in the first column are extracted from the tilt series, serving as the ground truth for comparison. (F) and $(\mathrm{G})$ are comparisons of the FRC curves of reprojections against the ground truth as depicted in (E) when $0^{\circ}$ and $45^{\circ}$ tilt is excluded in the leave-one-out test, respectively. 


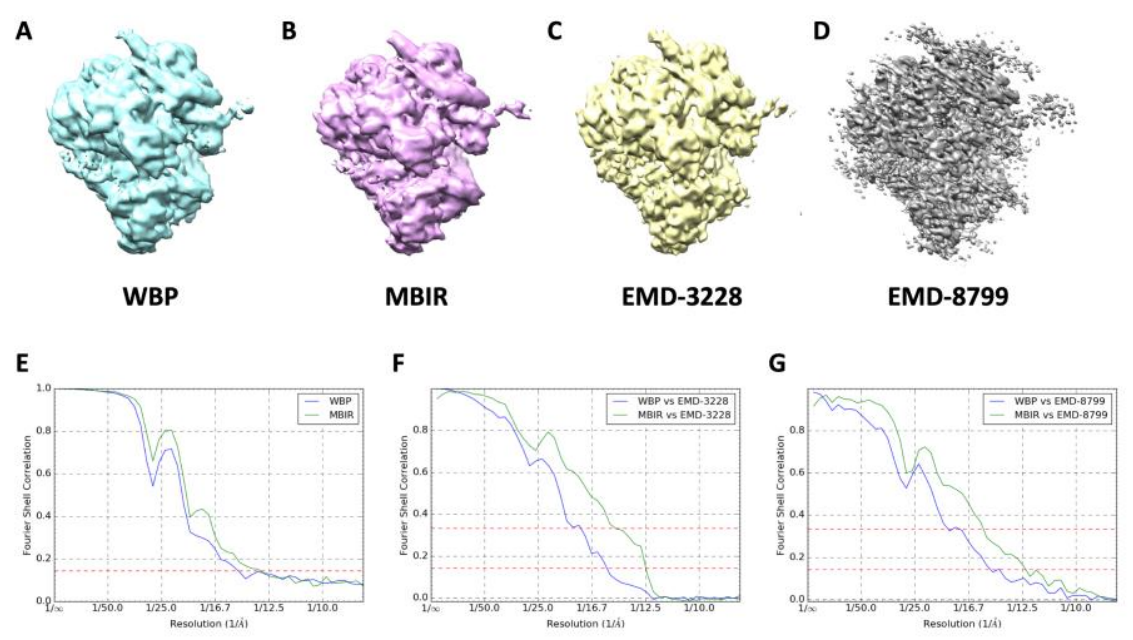

Figure 2. Comparison of subtomogram averages. (A) The averaged map of WBP's subtomograms. (B) The averaged map of MBIR's subtomograms. (C) The published subtomogram average map EMD-3228. (D) The published subtomogram average map EMD-8799. (E) The comparison of the "gold standard" FSC curves between WBP and MBIR maps. (F) FSC curves between the reference map EMD-3228 in (C) and the averaged subtomograms (WBP in blue and MBIR in green). (G) FSC curves between the reference map EMD-8779 in (D) and the averaged subtomograms (WBP in blue and MBIR in green).

\section{References}

Bharat, T.A.M., Russo, C.J., Löwe, J., Passmore, L.A., Scheres, S.H.W., 2015. Advances in SingleParticle Electron Cryomicroscopy Structure Determination applied to Sub-tomogram Averaging. Structure 23, 1743-1753.

Cardone, G., Grünewald, K., Steven, A.C., 2005. A resolution criterion for electron tomography based on cross-validation. J. Struct. Biol. 151, 117-129.

Deng, Y., Chen, Y., Zhang, Y., Wang, S., Zhang, F., Sun, F., 2016. ICON: 3D reconstruction with "missing-information" restoration in biological electron tomography. J. Struct. Biol. 195, 100-112.

Gilbert, P., 1972. Iterative methods for the three-dimensional reconstruction of an object from projections. Journal of Theoretical Biology. https://doi.org/10.1016/0022-5193(72)90180-4

Herman, G.T., 2009. Fundamentals of Computerized Tomography: Image Reconstruction from Projections. Springer Science \& Business Media.

Himes, B.A., Zhang, P., 2018. emClarity: software for high-resolution cryo-electron tomography and subtomogram averaging. Nat. Methods 15, 955-961.

Lucić, V., Förster, F., Baumeister, W., 2005. Structural studies by electron tomography: from cells to molecules. Annu. Rev. Biochem. 74, 833-865.

Radermacher, M., n.d. Weighted Back-projection Methods. Electron Tomography. https://doi.org/10.1007/978-0-387-69008-7_9

Venkatakrishnan, S.V., Drummy, L.F., Jackson, M., De Graef, M., Simmons, J., Bouman, C.A., 2015. Model-Based Iterative Reconstruction for Bright-Field Electron Tomography. IEEE Transactions on Computational Imaging. https://doi.org/10.1109/tci.2014.2371751 
Yan, R., Venkatakrishnan, S.V., Liu, J., Bouman, C.A., Jiang, W., 2019. MBIR: A cryo-ET 3D reconstruction method that effectively minimizes missing wedge artifacts and restores missing information. J. Struct. Biol. 206, 183-192. 